\title{
Multifaceted Immune Responses to African Swine Fever Virus: Implications for Vaccine Development
}

\author{
Tao Wang ${ }^{1}$, Yuan Sun ${ }^{1}$, S.J. Huang ${ }^{2}$, and Hua-Ji Qiu ${ }^{1}$ \\ ${ }^{1}$ Chinese Academy of Agricultural Sciences Harbin Veterinary Research Institute \\ ${ }^{2}$ Foshan University School of Life Science
}

May 15, 2020

\begin{abstract}
African swine fever (ASF) is a highly contagious, often fatal viral disease caused by African swine fever virus (ASFV), leading to severe hemorrhages in domestic pigs and wild boars. In 2007, ASF was reintroduced into Europe. Since then, ASF has spread to many European and Asian countries and now becomes a major concern to the swine industry worldwide. There have been various vaccine attempts, but no commercial ASF vaccines are available so far. A key hurdle in developing a safe and effective ASF vaccine is the limited understanding of the mechanisms of immune responses to and immune protection against ASFV infection. Some vaccine candidates show promising results, but more important scientific challenges remain unsolved. Here, we provide an overview of the current knowledge in innate and adaptive immune responses elicited by ASFV infection or different kinds of vaccine candidates. Additionally, the applications and prospects of vaccine candidates are discussed. Finally, we highlight the implications of these mechanisms for rational design of ASF vaccines.
\end{abstract}

\section{Hosted file}

manuscript-Transboundary and Emerging Diseases-20200515.doc available at https://authorea. com/users/322422/articles/451389-multifaceted-immune-responses-to-african-swine-fevervirus-implications-for-vaccine-development 


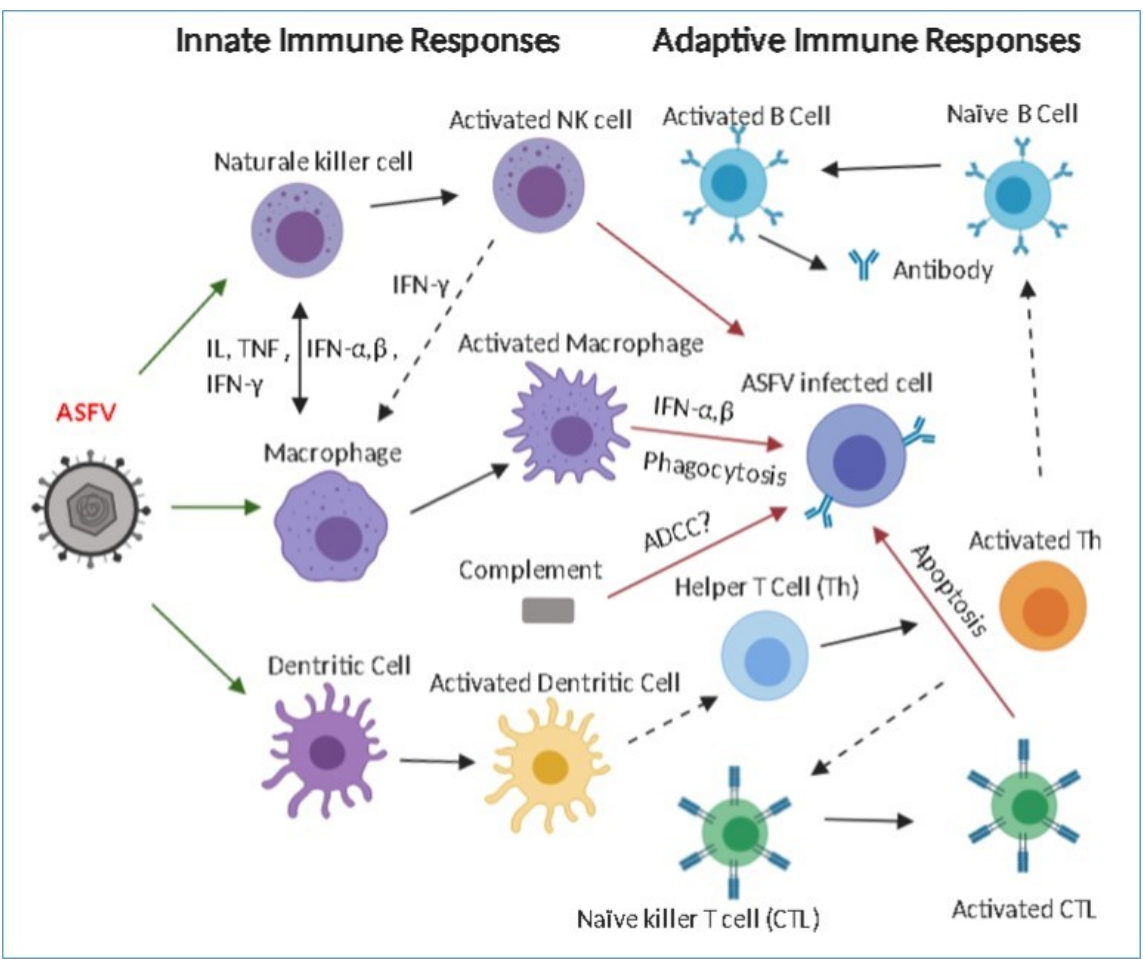

Approaches to African swine fever virus vaccine Development

a. Whole inactivated

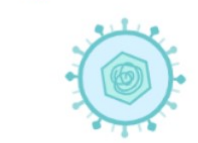

$0 \%$

b. Naturally attenuated strains OURT88/3 and NH/P68)

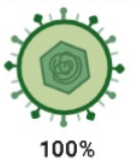

c. Adaptation of cell lines attenuated strain (BA71V, ASFV-G/VP110)

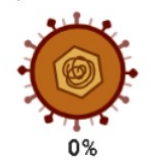

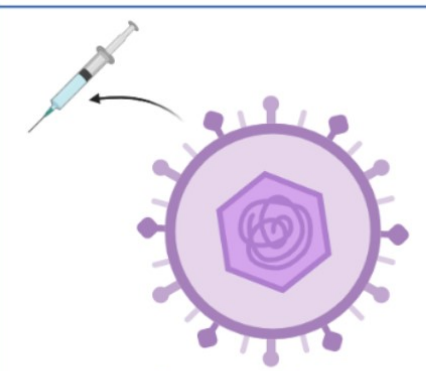

African swine fever virus

d. Gene deleted attenuated strains (CD2v, 9GI, Uk, DP148R, 1177L)

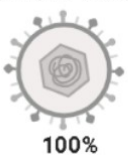

g. Recombinant viral vectors

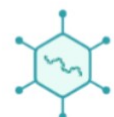

0/Not avaiable

f. Recombinant subunits (P30、P72、P54、CD2v)

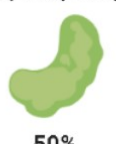

e. DNA or RNA

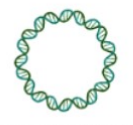

